\title{
Loading conditions of the knee: what does it mean?
}

\author{
Roland Becker $\cdot$ Sebastian Kopf $\cdot$ Jon Karlsson
}

Published online: 26 October 2013

(C) Springer-Verlag Berlin Heidelberg 2013

Bracing, for unloading the knee on healthy subjects, was studied in the leading article of the current issue by Orishimo et al. [12]. Loading or unloading the knee is a very interesting and important aspect when postsurgical treatment of cartilage or meniscal lesions is discussed.

Research about knee loading has been of interest for more than 30 years using finite element models [2], force plates [5] and in vivo technology [15]. Some very interesting in vivo data have shown that walking causes femorotibial peak forces of $261 \%$ body weight and descending stairs up to $346 \%$ of body weight [7]. Knee loading can increase up to $550 \%$ body weight during sudden balance loss, showing the importance of the interaction of the muscles. It is well known that the medial compartment takes significantly more load than the lateral one, which is on average $60-70 \%$ of the load during level walking. Loading can rise significantly in osteoarthritic knees $[6,8]$.

A minor change in leg alignment shifts the load to the medial or lateral compartment. This has been known for many years and has been the basis for internal or external correction of the leg alignment $[1,13]$.

\section{R. Becker $(\square)$}

Department of Orthopaedic and Traumatology, City Hospital Brandenburg, Hochstrasse 29, 14770 Brandenburg/Havel, Germany

e-mail: roland_becker@yahoo.de

\section{S. Kopf}

Center for Musculoskeletal Surgery, Charite', University

Medicine, Berlin, Germany

e-mail: Sebastian.Kopf@charite.de

\section{J. Karlsson}

Department of Orthopaedics, Sahlgrenska University Hospital, Molndal, Sweden

e-mail: jon.kssta@gmail.com
Gait analyses have shown that the adduction moment depends more on leg alignment rather than the degree of OA [4]. The adduction moment serves as the surrogate for medial compartment load.

Despite the strong correlation between the mechanical axis and the adduction moment, other factors such as increased rearfoot eversion, rearfoot internal rotation and forefoot inversion cause reduction in loading of the medial compartment [9]. Thus, it seems to make sense to perform a "brace-test", for instance, before high tibial osteotomy is considered in osteoarthritic knees. By using a brace in $4^{\circ}$ and $8^{\circ}$ valgus in osteoarthritic knees, the reduction in medial tibial loading was 24 and $30 \%$, respectively [8]. However, this data seem to depend on the type of brace.

As previously mentioned, Orishimo et al. [12] performed their study on healthy subjects and not on patients suffering from osteoarthritis. Increased valgus stress decreased the adduction moment by $32 \%$. These data are comparable to findings in osteoarthritic patients. However, what would the clinical relevance be of lowering the adduction moment by 20 or $30 \%$ ? How much unloading of a compartment would be required to improve clinical symptoms, allow cartilage regeneration and or meniscus healing?

The current guidelines of postsurgical knee loading after cartilage repair, meniscus surgery or osteotomy are mainly based on clinical experience. However, there is published data which, for example show that by decreasing the adduction to $<2.5 \%$ body weight $\times$ height in patients after high tibial osteotomy showed better clinical outcome compared to patients without bracing [14].

How much correction do we need in order to not only relieve pain but also prevent progression of femorotibial OA? Many authors refer in clinical and basic science studies to the Fujisawa-point, a point where the mechanical 
axis crosses the tibial plateau [3]. This point is between 60 and $70 \%$ considering the lateral border of the plateau is $0 \%$ and the medial one $100 \%$. In this study, patients presented with the best clinical outcome when corrected into this range. A recent study by Lind et al. showed that a correction of the mechanical axis of the leg to $62.5 \%$ of the width of the tibia plateau lead to a decreased adduction moment of $2.8 \%$ body weight $\times$ height [10]. Though, a correction between 60 and $70 \%$ seems to make sense taking the clinical and biomechanical data into consideration.

However, we do not know exactly how much overloading of the contralateral, non-affected compartment will accelerate the degeneration. Also, we do not know exactly how much unloading might prevent or delay further degeneration or even how much loading or unloading cartilage can tolerate or require?

Unloading of the knee for 2 months using two monotube external distractors showed clinical improvement and cartilage regeneration in young patients suffering from osteoarthritis [16]. The minimal joint space width increased significantly from 1.1 to $1.7 \mathrm{~mm}$ and remained at that level for up to 2 years.

By contrast, in vitro studies have shown that biological and biomechanical properties of human chondrocytes seeded in collagen type I hydrogel were significantly increased when cyclic compressive loading was applied [11]. This shows that cartilage requires loading in order to maintain its intrinsic capability to sustain load.

On the one hand, we need knee loading in order to have durable cartilage, but on the other hand, we need some kind of unloading in order to allow healing and repair of tissue.

Better understanding of knee loading may help us to find more biological ways to treat these kinds of knee pathologies.

\section{References}

1. Bourne RB, Finlay JB, Papadopoulos P, Rorabeck CH, Andreae P (1984) In vitro strain distribution in the proximal tibia. Effect of varus-valgus loading in the normal and osteoarthritic knee. Clin Orthop Relat Res 18:285-292

2. Donahue TL, Hull ML, Rashid MM, Jacobs CR (2002) A finite element model of the human knee joint for the study of tibiofemoral contact. J Biomech Eng United States 124:273-280
3. Fujisawa Y, Masuhara K, Shiomi S (1979) The effect of high tibial osteotomy on osteoarthritis of the knee. An arthroscopic study of 54 knee joints. Orthop Clin North Am 10:585-608

4. Hurwitz D, Ryals A, Case J, Block JA, Andriacchi T (2002) The knee adduction moment during gait in subjects with knee osteoarthritis is more closely correlated with static alignment than radiographic disease severity, toe out angle and pain. J Orthop Res 20:101-107

5. Johnson F, Leitl S, Waugh W (1980) The distribution of load across the knee. A comparison of static and dynamic measurements. J Bone Joint Surg Br 62:346-349

6. Kumar D, Manal KT, Rudolph KS (2013) Knee joint loading during gait in healthy controls and individuals with knee osteoarthritis. Osteoarthr Cartil 21:298-305

7. Kutzner I, Heinlein B, Graichen F, Bender A, Rohlmann A, Halder A, Beier A, Bergmann G (2010) Loading of the knee joint during activities of daily living measured in vivo in five subjects. J Biomech 43:2164-2173

8. Kutzner I, Küther S, Heinlein B, Dymke J, Bender A, Halder AM, Bergmann G (2011) The effect of valgus braces on medial compartment load of the knee joint-in vivo load measurements in three subjects. J Biomech 44:1354-1360

9. Levinger P, Menz HB, Morrow AD, Bartlett JR, Feller JA, Bergman NR (2013) Relationship between foot function and medial knee joint loading in people with medial compartment knee osteoarthritis. J Foot Ankle Res 6:33

10. Lind M, McClelland J, Wittwer JE, Whitehead TS, Feller JA, Webster KE (2013) Gait analysis of walking before and after medial opening wedge high tibial osteotomy. Knee Surg Sports Traumatol Arthrosc Germany 21:74-81

11. Nebelung S, Gavenis K, Lüring C, Zhou B, Mueller-Rath R, Stoffel M, Tingart M, Rath B (2012) Simultaneous anabolic and catabolic responses of human chondrocytes seeded in collagen hydrogels to long-term continuous dynamic compression. Ann Anat 194:351-358

12. Orishimo KF, Kremenic IJ, Lee SJ, McHugh MP, and Nicholas SJ (2012) Is valgus unloader bracing effective in normally aligned individuals: implications for post-surgical protocols following cartilage restoration procedures. Knee Surg Sports Traumatol Arthrosc 1-6

13. Paley D, Tetsworth K (1992) Mechanical axis deviation of the lower limbs. Preoperative planning of uniapical angular deformities of the tibia or femur. Clin Orthop Relat Res 280:48-64

14. Prodromos CC, Andriacchi TP, Galante JO (1985) A relationship between gait and clinical changes following high tibial osteotomy. J Bone Joint Surg Am 67:1188-1194

15. Taylor WR, Heller MO, Bergmann G, Duda GN (2004) Tibiofemoral loading during human gait and stair climbing. J Orthop Res United States 22:625-632

16. Wiegant K, van Roermund PM, Intema F, Cotofana S, Eckstein F, Mastbergen SC, Lafeber FP (2013) Sustained clinical and structural benefit after joint distraction in the treatment of severe knee osteoarthritis. Osteoarthr Cartil. doi:10.1016/j.joca.2013.08. 006 